\title{
(2) Structural evaluation in inherited retinal diseases
}

\section{OPEN ACCESS}

\author{
Malena Daich Varela (D) ,', Burak Esener (D) ,' Shaima A Hashem, 1,2 \\ Thales Antonio Cabral de Guimaraes (D) , 1,2 Michalis Georgiou (D) , 1,2 \\ Michel Michaelides ${ }^{1,2}$
}

${ }^{1}$ Moorfields Eye Hospital City Road Campus, London, UK ${ }^{2} \mathrm{UCL}$ Institute of Ophthalmology, University College London, London, UK ${ }^{3}$ Department of Ophthalmology, Inonu University School of Medicine, Malatya, Turkey

\section{Correspondence to}

Michel Michaelides, University College London, London EC1v 2D, UK

michel.michaelides@ucl.ac.uk

Received 5 March 2021

Revised 7 April 2021

Accepted 21 April 2021
Check for updates

(c) Author(s) (or their employer(s)) 2021. Re-use permitted under CC BY. Published by BMJ.

To cite: Daich Varela M, Esener B, Hashem SA, et al. Br J Ophthalmol Epub ahead of print: [please include Day Month Year]. doi:10.1136/ bjophthalmol-2021-319228

\begin{abstract}
Ophthalmic genetics is a field that has been rapidly evolving over the last decade, mainly due to the flourishing of translational medicine for inherited retinal diseases (IRD). In this review, we will address the different methods by which retinal structure can be objectively and accurately assessed in IRD. We review standard-of-care imaging for these patients: colour fundus photography, fundus autofluorescence imaging and optical coherence tomography (OCT), as well as higher-resolution and/or newer technologies including OCT angiography, adaptive optics imaging, fundus imaging using a range of wavelengths, magnetic resonance imaging, laser speckle flowgraphy and retinal oximetry, illustrating their utility using paradigm genotypes with on-going therapeutic efforts/trials.
\end{abstract}

\section{INTRODUCTION}

Inherited retinal diseases (IRD) are a heterogeneous group of conditions, with unrivalled phenotypic and genotypic variability. This group includes rod-cone, cone-rod, isolated cone and macular dystrophies (MD), and cone and rod dysfunction syndromes. ${ }^{1-5}$ Approximately 1 in 2000 individuals worldwide are affected by this group of disorders, and 2.7 billion people are healthy carriers of at least one likely disease-causing variant linked to autosomal recessive IRD ${ }^{6}$; with IRD being the most common cause of blindness in the working age population in England. $^{7}$

The huge disease burden caused by $\operatorname{IRD}^{8}$ and the advancements in retinal genetics, imaging and molecular biology, have led to the development of clinical trials of novel therapeutics. ${ }^{9-11}$ Gene supplementation, gene editing through clustered regularly interspaced short palindromic repeats (CRISPR) technology, antisense oligonucleotides, optogenetics and stem cell-based therapies are some of the techniques currently being tested to improve eyesight and/or reduce the rate of disease progression. ${ }^{1-5} 1012$ The multiple on-going and upcoming trials emphasise the need for reliable and repeatable measurements, for both patient stratification and endpoint assessment. Structural evaluation is of paramount importance, since most treatments target specific cell populations and aim to halt degeneration or restore retinal structure, along with clinically meaningful functional improvement or preservation of visual function.

Multi-modal assessment of retinal architectural integrity is employed to explore disease natural history, monitor progression, inform advice on prognosis, elucidate disease pathogenesis, stratify patients and evaluate the effect of therapies. Herein, we will review the different methods for structural assessment, focusing on specific genotypic paradigms with on-going therapeutic efforts (table 1).

\section{Colour fundus photography}

Colour fundus photography (CFP) is a widely available tool to document the retinal appearance. To begin with, drawings were used for this purpose and around the end of the 19th century, fundus cameras started to become available, with constant evolution ever since, including a broad range of digital and widefield options-arguably the two most important developments. A basic feature of retinal cameras is their optical angle of view; ranging from $20^{\circ}$ (particularly used to image the optic disc), $30^{\circ}$ (standard retinal view), wider angles such as $45^{\circ}$ and $60^{\circ}$ and ultra-wide field covering $200^{\circ}$ (approximately $80 \%$ of the retina). ${ }^{13}$ These devices can also be classified based on the use of confocal optics or flash-based systems. Currently, the former is most efficient, suppressing scattered light and resulting in sharp, high contrast and high chromatic images. ${ }^{14}$ Different filters have also been developed to enhance particular structures. Red light improves visualisation of the choroid and its pattern; green light (red-free) is best for retinal vasculature, haemorrhages, drusen, exudates and the overall retina; and blue light is used to focus on the anterior retinal layers. ${ }^{15} \mathrm{CFP}$, and particularly ultra-wide field CFP, is almost universally included as part of both standard-of-care and research visits for trials and studies in IRD, since it facilitates both documentation, evaluation and monitoring of for example, progression of areas of atrophy, ${ }^{16}$ treated areas and retinotomy sites, inflammatory features including vasculitis, retinitis and choroiditis, ${ }^{17}$ and is also valuable for teaching. CFP is frequently used for topographical tracking of functional tests, such as fundus-guided microperimetry, and moreover, can be used to overlay a wide array of functional assessments onto the retinal landscape.

\section{Fundus autofluorescence imaging}

Fluorophores are molecules that have the capacity to emit light when excited by appropriate wavelengths, a characteristic called autofluorescence (AF). ${ }^{18}$ Exogenous fluorophores such as fluorescein and indocyanine green are broadly used for diagnostic purposes in ophthalmology - with their application in angiography not discussed herein given their limited utility in IRD. Endogenous fluorophores like lipofuscin can be found in most eukaryotic cells, and in the eye are predominantly 
Table 1 Summary of the current and under development methods for IRD structural evaluation

\begin{tabular}{|c|c|c|}
\hline Imaging modality & Characteristics & Use in inherited retinal diseases (IRD) \\
\hline Colour fundus photography & $\begin{array}{l}\text { Classified based on the use of confocal optics or flash-based systems. Different } \\
\text { filters can be employed to enhance particular structures: red light } \rightarrow \text { choroid; } \\
\text { green light (red-free) } \rightarrow \text { retinal vasculature, drusen, exudates; blue light } \rightarrow \\
\text { anterior retinal layers. }\end{array}$ & $\begin{array}{l}\text { Fundamental tool that facilitates teaching, documentation, } \\
\text { evaluation and monitoring. }\end{array}$ \\
\hline Fundus autofluorescence imaging (FAF) & $\begin{array}{l}\text { Short wavelength-FAF (SW-FAF): macula appears hypoautofluorescent. Good } \\
\text { for evaluation of areas with photoreceptor loss but relatively intact retinal } \\
\text { pigment epithelium, and subretinal hyper-reflective material. } \\
\text { Near infrared-FAF (NIR-FAF): macula is hyperautofluorescent. It detects } \\
\text { geographic atrophy and pigment migration earlier than SW-FAF. }\end{array}$ & $\begin{array}{l}\text { Its property of revealing the retina's health and metabolism makes } \\
\text { it an important tool for diagnosing and monitoring IRD. It also } \\
\text { provides valuable insights on disease pathophysiology. }\end{array}$ \\
\hline Optical coherence tomography (OCT) & $\begin{array}{l}\text { Enables highly detailed qualitative and quantitative assessments of the retinal } \\
\text { layers. }\end{array}$ & $\begin{array}{l}\text { Key tool to accurately monitor anatomical changes. Also employed } \\
\text { intraoperatively in gene therapy clinical trials. }\end{array}$ \\
\hline OCT angiography & $\begin{array}{l}\text { Provides tri-dimensional visualisation of the retinal microvasculature and capillary } \\
\text { plexi. }\end{array}$ & $\begin{array}{l}\text { Useful to identify choroidal neovascularisation in association with } \\
\text { IRD. }\end{array}$ \\
\hline Adaptive optics & $\begin{array}{l}\text { Two types: (i) confocal is used to resolve the cone and perifoveal rod mosaics; (ii) } \\
\text { non-confocal (split detection) identifies cones with abnormal outer segments. }\end{array}$ & $\begin{array}{l}\text { Enables non-invasive cellular imaging. Helpful to increase our } \\
\text { understanding of IRD. Also used for monitoring progression and in } \\
\text { research settings. }\end{array}$ \\
\hline Optoretinography & $\begin{array}{l}\text { Allows mapping of stimulus-evoked functional intrinsic optical signal using near } \\
\text { infrared light. }\end{array}$ & $\begin{array}{l}\text { May be useful for assessing photoreceptor integrity and } \\
\text { dysfunction (still under development). }\end{array}$ \\
\hline Laser speckle flowgraphy & $\begin{array}{l}\text { Employs the laser speckle phenomenon to quantify in vivo the circulation in the } \\
\text { optic nerve head, choroid and retina. }\end{array}$ & $\begin{array}{l}\text { Has been used to correlate blood flow with other structural and } \\
\text { functional parameters in IRD. }\end{array}$ \\
\hline Retinal oximetry & Measures oxygen metabolism by capturing how haemoglobin absorbs light. & $\begin{array}{l}\text { May represent an alternative way to assess outer retinal } \\
\text { degeneration in IRD (still under development). }\end{array}$ \\
\hline Functional magnetic resonance imaging & Provides high resolution imaging of the brain including the visual cortex. & $\begin{array}{l}\text { Useful to assess plasticity and remodelling following visual field } \\
\text { defects, congenital visual impairment and/or interventions. }\end{array}$ \\
\hline
\end{tabular}

in the retinal pigment epithelium (RPE). ${ }^{19}$ The AF signal corresponds to the concentration of lipofuscin and other secondary fluorophores, which also relates to the pace at which photoreceptor outer segments (OS) are metabolised by the RPE cells. ${ }^{20}$

There are two main techniques of fundus autofluorescence (FAF) imaging: (i) short wavelength AF (SW-FAF), acquired with a $488 \mathrm{~nm}$ blue light that excites lipofuscin and N-retinylidene-Nretinylethanolamine (A2E), with an emission range between 560 and $700 \mathrm{~nm}$; and (ii) near infrared AF (NIR-FAF), where excitation occurs at $787 \mathrm{~nm}$ (thereby also exciting melanin located in the RPE and choroid) and emission around $800 \mathrm{~nm} .{ }^{19}{ }^{21}$ Both employ a confocal scanning laser ophthalmoscope (SLO) and with both the optic nerve and vessels appear dark due to lack of lipofuscin and light absorption by blood, whereas the macular appearance differs. ${ }^{22}$ In SW-FAF, the macular region is hypoautofluorescent, while with NIR-FAF, it is hyperautofluorescent relative to the surrounding retina. ${ }^{22}$ These two imaging techniques complement each other, as NIR-FAF detects geographic atrophy and pigment migration earlier than SW-FAF, while the latter is better at detecting areas with photoreceptor loss but intact RPE, and subretinal hyper-reflective material. ${ }^{23}$

Hypoautofluorescence can be due to a reduced concentration of lipofuscin (eg, $\mathrm{RDH} 5$-fundus albipunctatus, figure 1A, and RPE65-early onset severe retinal dystrophy (EOSRD)), ${ }^{24} 25$ RPE atrophy (eg, choroideraemia, figure 1B), ${ }^{26}$ fibrotic tissue (eg, late-stage BEST1 macular dystrophy, figure $1 \mathrm{C})^{27}$ or signal
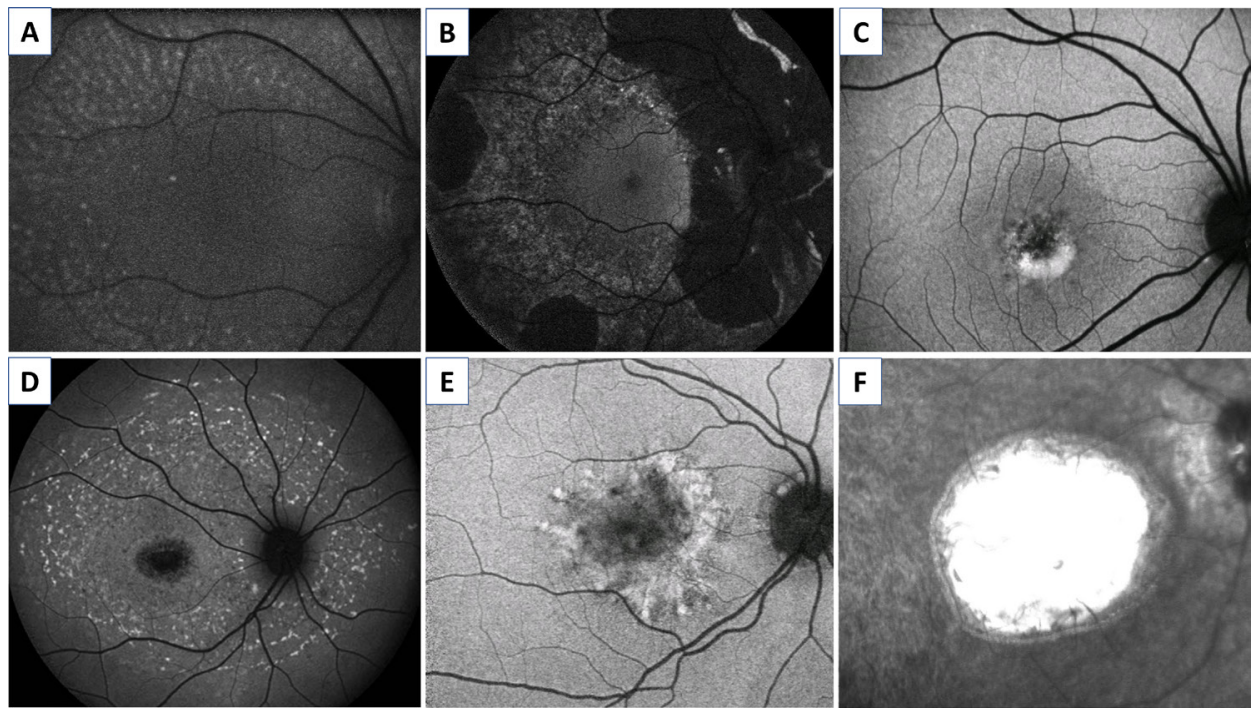

Figure 1 Examples of fundus autofluorescence patters in inherited retinal disease. Hypoautofluorescent defects secondary to: (A) reduced concentration of lipofuscin in $R D H 5$-fundus albipunctatus, (B) retinal pigment epithelium atrophy in choroideraemia and (C) fibrotic tissue in latestage BEST1 vitelliform macular dystrophy. Hyperautofluorescent defects secondary to: (D) increase in lipofuscin appearing as flecks in $A B C A 4-$ retinopathy, (E) drusen in EFEMP1-autosomal dominant drusen and (F) window defect in NMNAT1-Leber congenital amaurosis. (A-E) Shortwavelength and (F) near infrared autofluorescence images. 
absorption by cells or extracellular material overlying the RPE (eg, subretinal bleb following gene therapy administration). ${ }^{28}$ Hyperautofluorescence, on the other hand, can be explained by an increase in lipofuscin (eg, flecks/vitelliform deposition in ABCA4/BEST1/PRPH2-associated retinopathy, figure 1D), ${ }^{29}$ intraretinal fluid (cystoid macular oedema), ${ }^{30}$ drusen (eg, EFEMP1-autosomal dominant drusen, figure $1 \mathrm{E})^{1}$ and window defects (absence of signal blockage, eg, macular dysplasia in NMNAT1-Leber congenital amaurosis (LCA), figure 1F). ${ }^{931} 32$

FAF imaging has become a key tool to diagnose and monitor the progression of IRD, because of its property of revealing the retina's health and metabolism. It has also provided valuable insights into disease pathophysiology. Generalised lack of $\mathrm{AF}$ in patients with EOSRD/LCA has typically been associated with variants in genes that affect the visual cycle, such as RPE65 and LRAT. ${ }^{33}$ Nevertheless, the universal nature of this feature has been recently questioned, with retained AF shown in some patients with RPE65-retinopathy, mainly at the posterior pole. ${ }^{25}$ Hypoautofluorescent lesions have also been of interest, with their intensity varying according to the depth of the outer layer defect. Using semiautomated software, the decreased AF can be graded into definitely decreased or questionably decreased, taking as reference the hypoautofluorescence of the optic nerve head, and the rate of atrophy enlargement tracked accurately over time. ${ }^{3-38}$ This has been investigated in detail in multiple observational Stargardt disease (STGD) studies, and is being employed currently as an endpoint in interventional trials. ${ }^{36-39}$ In direct contrast, variants in genes that affect the clearance of all-trans-retinal from the interior of the outer segment discs, increase the accumulation of lipofuscin and related fluorophores, resulting in hyperautofluorescence. This characteristically occurs in ABCA4- and PRPH2-associated retinopathy and also underlies the perifoveal high intensity ring seen in a broad range of IRD. ${ }^{1041}$ The latter annular pattern of increased signal has been associated with the boundary where the ellipsoid zone (EZ) starts becoming discontinuous, and also with decreased retinal sensitivity (assessed by multifocal electroretinography and fine matrix mapping). ${ }^{42}{ }^{43}$ It is likely to represent ongoing RPE/ photoreceptor stress and an intermediate stage before cell loss. The ring can be present in both cone-rod dystrophies (CORD) and rod-cone dystrophies (RCD), and by serially measuring (eg, area or greatest linear dimension) either its expansion in CORD, or constriction in RCD, disease progression can be quantified. ${ }^{44}$ The ring can be seen both with SW-FAF and NIR-FAF, with the caveat of being smaller in the latter. It has been postulated that this phenomenon may indicate that NIR detects earlier cellular changes, that will later become visible with SW-FAF. ${ }^{45}$

Qualitative assessment of the area of decreased AF is also useful for longitudinal assessment of patients with RPE atrophy. ${ }^{46}$ The decrease in signal has been correlated with the loss of RPE, and has been proposed as a metric in MD such as STGD, and cone dystrophies (COD)/CORD. ${ }^{39} 47$ In conditions such as choroideraemia and RCD, where there is conserved macular signal due to relatively preserved structure, quantification of the area of intact signal can be a meaningful measurement of disease progression or prevention of degeneration, clinically and in clinical trials, respectively. ${ }^{44} 48$ Ultra-wide field FAF patterns are also increasingly being used to categorise conditions such as RCD and STGD, including identifying the magnitude and extent of midperipheral and far-peripheral retinal involvment. ${ }^{46}$

A SW-SLO system using a $450 \mathrm{~nm}$ blue light has been used to image the retina, providing pictures that are referred to as 'colour-FAF'. ${ }^{49}$ The emission spectrum can be subdivided into two images: red $(560-700 \mathrm{~nm})$ and green $(510-560 \mathrm{~nm})$. This technique provides additional information about minor fluorophores such as advanced glycation end products and oxidised fluorescent form flavin adenine dinucleotide (FAD) of the redox pair FAD-FADH2, that appear on the green, short wavelength image. ${ }^{50}$ Lipofuscin and A2E are mostly responsible for long wavelength emission (red image). One application for green AF has been to monitor subretinal hyper-reflective material over Bruch's membrane,$^{50}$ and in the characterisation of small, central lesions, given its lower absorption by macular pigments. ${ }^{51}$ Another tested approach is the use of SW reduced-illuminance $\mathrm{AF}$, which employs a custom percentage of the laser power and can go as low as $25 \%$ of conventional intensity. ${ }^{52}$ Studies have shown a high correlation with standard $\mathrm{AF}$ in patients with STGD, but with better tolerability-bearing in mind the potential toxicity of higher intensities for patients with IRD. ${ }^{53}$

Beyond standard FAF, fluorescence lifetime imaging ophthalmoscopy (FLIO) is a developing modality for further functional imaging, based on the decay time of the fluorescent molecules. ${ }^{54}$ FLIO is a promising tool to detect and assess varying metabolic states of the retina, potentially allowing characterisation of disease areas before damage is visible with other structural imaging methods. It also enables differentiation between zones with preserved outer layers, photoreceptor loss and photoreceptor-RPE complex disruption. Hyperfluorescent FLIO rings with short FAF lifetimes may provide insight into the pathophysiological status of RCD-affected retinas, perhaps providing a more detailed/sensitive assessment of disease progression. ${ }^{55}$

\section{Optical coherence tomography}

Since its introduction in 1988, optical coherence tomography (OCT) has become the most valuable tool for retinal structural assessment, providing an in vivo cross-sectional view of the retina that has revolutionised clinical and academic practice. ${ }^{56}$ Initially, the signals were time-encoded and these devices were referred to as time-domain OCT. ${ }^{57}$ Later on, spectral-domain OCT provided an improved axial resolution (from 10 to $2 \mu \mathrm{m}$ ) and faster acquisition speed, by collecting backscattering signals through a broad-bandwidth light source. ${ }^{58}$ More recently, swept-source OCT using rapidly tunable lasers with longer wavelength, has allowed imaging of deeper structures, improved visualisation even with media opacity, higher contrast and wider scans. ${ }^{5960}$ The higher resolution of OCT not only enabled qualitative assessment of multiple retinal layer integrity, but moreover, allowed repeatable quantitative/volumetric measurements. In IRD, OCT has transformed disease characterisation, including revealing countless phenotypic features such as retinal tubulations at the margin of the outer retinal loss in choroideraemia and other advanced retinal dystrophies, ${ }^{6162}$ intraretinal foveal schisis in X-linked retinoschisis, ${ }^{63}$ and thick, abnormally laminated retina in CRB1-associated disease. ${ }^{64}$

The ability to accurately determine anatomical degeneration has changed the IRD landscape. The shortening of photoreceptor OS is one of the earliest findings in RCD and can be assessed by measuring the length between the inner surface of the hyperreflective layer between inner segments and OS of photoreceptors, also known as the EZ, and the inner surface of the RPE .${ }^{6566}$ The shortening of this layer has been shown to significantly correlate with several functional parameters including visual field, central retinal sensitivity and best-corrected visual acuity (BCVA) ${ }^{67-69}$ Arguably more robust metrics include serial EZ area (EZA) and diameter (EZW, width), which have been explored extensively as measurements in RP and LCA monitoring and are being used currently as structural endpoints in clinical trials 

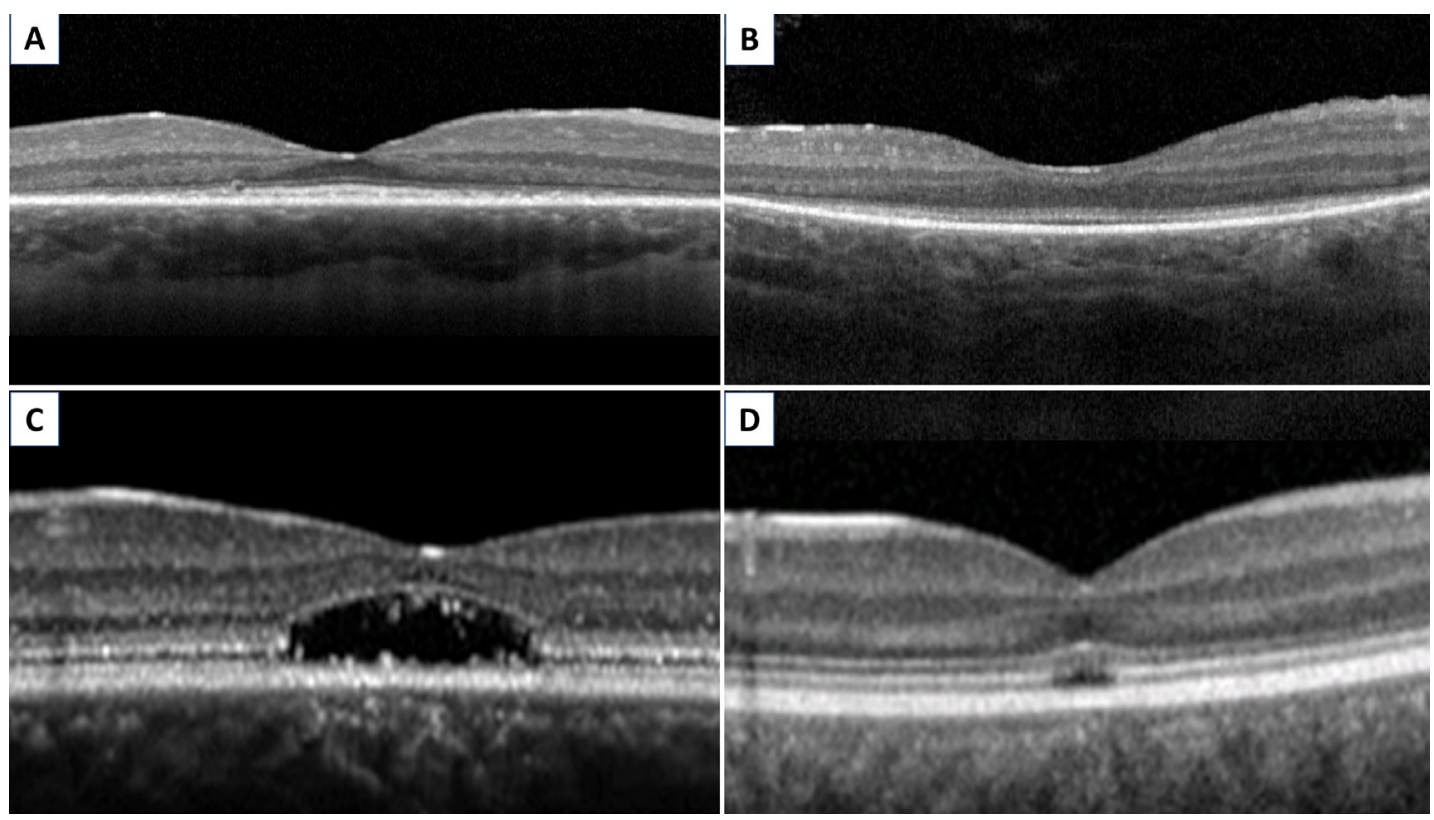

Figure 2 Optical coherence tomography evaluation of the ellipsoid zone (EZ). Foveal EZ preservation, with peripheral EZ loss, in (A) RPGR-RP and (B) RPE65-LCA. Foveal EZ loss, with peripheral EZ preservation, in (C) ABCA4-MD and (D) CNGB3-ACHM. RP: retinitis pigmentosa; LCA: Leber congenital amaurosis; ACHM, achromatopsia; MD, macular dystrophies.

(figure 2A,B). ${ }^{25} 447071$ In MD and COD/CORD, quantification of EZA and EZW loss can also serve as eligibility criteria and outcome measurements for intervention (figure 2C,D). ${ }^{72} \mathrm{EZA} /$ EZW are metrics of great value for elucidating disease natural history in IRD. EZA loss has been shown to be greater than the area of decreased AF in STGD. ${ }^{47273}$ These observations support the theory that photoreceptor degeneration precedes RPE loss in STGD, or that functional RPE loss precedes the structural loss of RPE leading to photoreceptor loss before structural damage becomes apparent on FAF-importantly, this is in direct contrast to prevailing pathogenesis descriptions alluding to RPE loss preceding photoreceptor degeneration, ${ }^{74}$ and may thereby necessitate a paradigm shift.

Other useful OCT features are volumetric analysis and thickness assessments. Parameters such as central foveal thickness, submacular choroidal thickness (measured with standard and enhanced depth imaging) and macular volume are significantly reduced in patients with RCD, when compared with normal controls. ${ }^{75}$ However, these latter parameters are not always correlated with visual function, thereby measuring the EZ remains one of the most sensitive ways of tracking the progression of RCD. ${ }^{76}$ In addition, outer nuclear layer-foveal thickness has been frequently used as a surrogate measurement of foveal cone number, even though studies employing adaptive optics cellular imaging have proved the lack of correlation between these two parameters in conditions such as achromatopsia (ACHM). ${ }^{77-79}$

A present challenge regarding OCT analysis in patients with IRD (particularly CORD and STGD) is that automated retinal layer segmentation tends to be unreliable when retinal architecture is altered, requiring time consuming manual correction. ${ }^{80}$ To solve this issue, semiautomated segmentation methods have been developed, based on the selection and manipulation of a subset of scans. ${ }^{80}$ These methods may provide reliable measurements within particular Early Treatment Diabetic Retinopathy Study (ETDRS) rings, resulting in relevant diagnosis and monitoring information. ${ }^{81}$ However, inaccuracies remain regarding segmentation of the outer retina and atrophic areas, especially in the outer ETDRS ring, which still require manual correction. ${ }^{82}$ Deep learning approaches will likely be helpful in the near future to decrease the need for costly and impractical manual correction.

OCT can also be employed intraoperatively to facilitate optimal targeting and safe treatment delivery in subretinal injections of gene therapy products in IRD. ${ }^{84}$

\section{OCT angiography}

OCT angiography (OCTA) provides tri-dimensional visualisation of retinal microvasculature and capillary plexi. ${ }^{85}$ It has become an easier, faster and safer alternative to fluorescein and indocyanine green angiography; although not entirely replacing angiography, which can unlike OCTA, demonstrate leakage. OCTA is particularly helpful in identifying choroidal neovascularisation in association with vitelliform deposition in IRD. Multiple studies analysing the central 3 and $12 \mathrm{~mm}$ have shown decreased perfusion and vessel length density in the superficial and deep plexi of the choriocapillaris in patients with RCD. ${ }^{86-88}$ The loss of photoreceptors causes a reduction in the retina's oxygen consumption, thereby likely leading to the aforementioned vascular changes. ${ }^{89}$ A significant association between these and other features, such as the width and integrity of the EZ, BCVA and visual field has been demonstrated. ${ }^{86-88} 90$ Recently, reduced microvascular density has been associated with a decreased number of cones, quantified with adaptive optics imaging. ${ }^{91}$ It has also been postulated that the observed decline of choroidal vessel density occurs at late stages of retinal degeneration and further aggravates photoreceptor dysfunction. Therefore, analysing the integrity of the choroidal vasculature may be important in predicting the progression of IRD, as well as the responsiveness to treatment. ${ }^{92}$

\section{Adaptive optics}

Adaptive optics scanning light ophthalmoscopy (AOSLO) allows for non-invasive cellular imaging, thereby helping to improve our understanding of IRD. ${ }^{93}$ An increasing number of natural history studies and ongoing/planned interventional 


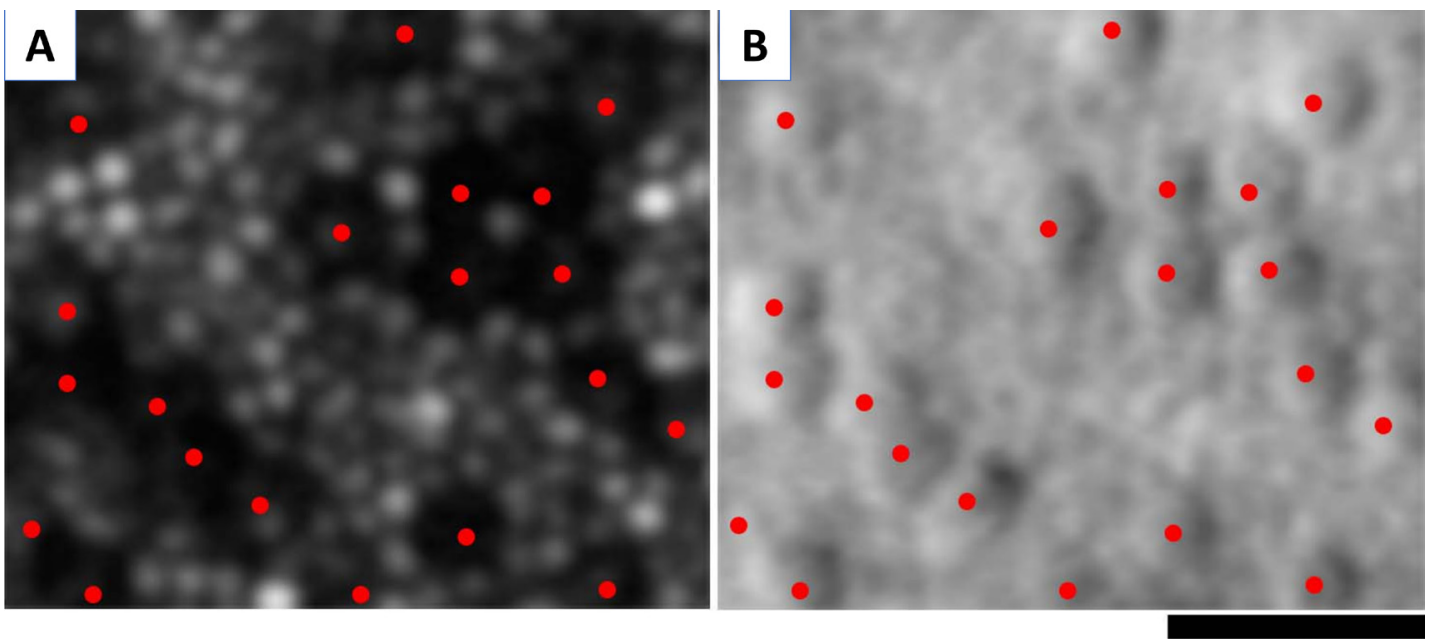

Figure 3 Cellular imaging with adaptive optics scanning light ophthalmoscopy (AOSLO). AOSLO imaging in CNGA3-associated achromatopsia: (A) confocal image, with red dots marking the 'dark' (non-waveguiding) cones. Cones are surrounded by waveguiding rods. (B) Non-confocal (split detection) image over the exact same region with overlying red dots showing the cones marked in $(A)$, which colocalise with cone inner segments, surrounded by rods. Scale bar: $20 \mu \mathrm{m}$.

clinical trials exploit AOSLO both for participant selection, stratification and monitoring treatment safety and efficacy. ${ }^{93-97}$ There are currently two main types of detection: (i) confocal, which uses the light that is backscattered by photoreceptors with relatively intact $O S$ to resolve the cone and perifoveal rod mosaics (figure 3A); and (ii) non-confocal (split detection), that processes images capturing the light to the right and left of the confocal aperture, enabling the identification of cones with abnormal OS (figure $3 \mathrm{~B}$ ). ${ }^{93}$ Several metrics are often employed, including cone density and spacing, peak cone density, Voronoi analysis of the regularity of the mosaic and reflectivity. ${ }^{98}$

AOSLO advanced retinal phenotyping has provided novel insights into IRD. It has been described that at $1^{\circ}$ from the fovea, a cohort of patients with CORD had 0.7 times greater spacing than a control group, and a cohort of patients with RCD, 0.2 times greater spacing. ${ }^{99}$ The more spacing between the cells, the less density of cones in that area. While it would be predicted that $\mathrm{AO}$ imaging and cone density would be abnormal in CORD, in RCD, one might expect there to be relatively preserved cone function and structure until advanced stages. However, AO imaging has shown that in patients with RCD (mean retinal sensitivity greater than $35 \mathrm{~dB}$ ), the cone density is decreased, even in areas where the EZ appears intact on OCT. Therefore, $\mathrm{AO}$ can monitor patients with mild RCD before changes are evident on OCT. ${ }^{100}$ Moreover, by detecting change more sensitively and thereby in shorter periods of times, AO may be helpful in determining the rate of progression in a far more timely fashion compared with other modalities. ${ }^{101}$ Qualitatively, cone-free patches have also been demonstrated at the fovea of patients with RCD, while these were absent in the normal population. ${ }^{99}$ Similarly, in STGD, AOSLO has shown early parafoveal decrease of photoreceptors. ${ }^{29}$ Nevertheless, despite the exquisite ultra-resolution of AO cellular imaging, it is significantly limited by the lack of a commercially available compact device and the inability to acquire standardised images in the majority of patients with IRD—in direct contrast to OCT.

However, AOSLO has been successfully employed in several deep-phenotyping studies for ACHM, showing highly variable residual cone density for $C N G A 3^{78}$ - and $C N G B 3^{77}$-ACHM (both genotypes have on-going gene therapy trials), better preserved mosaic in GNAT2 ${ }^{102}$-ACHM and greater degeneration in $A T F 6^{79}$ - and PDE6C ${ }^{40}$-ACHM. AOSLO cone counting with split-detection imaging has been reported to have good repeatably in STGD, RPGR-RCD and CNGA3- and CNGB3ACHM, with variability between different diseases. ${ }^{103} 104$ Furthermore, AOSLO can contribute to the differentiation between entities, such as oligocone trichromacy (sparse mosaic of normal wave-guiding cones at the fovea) and bradyopsia (relatively intact photoreceptor mosaic). ${ }^{105}$ Nonetheless, the correlation between $\mathrm{AO}$ images and functional parameters is still under evaluation; with AO-guided cellular psychophysical testing still in early development-and the promise of 'nanoperimetry'. Intriguingly, and with potential therapeutic implications, it has been reported that even with $40 \%$ of the normal cone density, BCVA and retinal sensitivity remain within normal limits. ${ }^{106}$

\section{Optoretinography}

Optoretinography (ORG) is a recent technique that allows mapping of stimulus-evoked functional intrinsic optical signal (IOS) using near infrared light-'functional imaging'. ${ }^{107}$ During phototransduction, a light stimulus causes slight shrinkage of photoreceptor OS by narrowing the inter-disc spacing, leading to bleaching and IOS (scattering and refractive index, among others). ${ }^{108}$ Measuring IOS has been correlated with photoreceptor integrity, which may, among other potential applications, have utility in stratification of patients for new therapies. ${ }^{109}$ ORG has been assessed in patients using high-speed OCT and AOSLO, although improved instruments and software is still required to optimise the technology. ${ }^{110-112}$ Preclinical studies have investigated ORG in murine models of photoreceptor dystrophy such as RCD, and shown that ORG was able to detect photoreceptor dysfunction. ${ }^{113}$ The perfecting of this modality may provide us with deeper evaluation of how photoreceptors function and respond to new therapies.

\section{Laser speckle flowgraphy}

Laser speckle flowgraphy (LSF) employs the laser speckle phenomenon to quantify in vivo the circulation in the optic 
nerve head, choroid and retina. ${ }^{114}$ In RCD, LSF has been used to show that decreased macular choroidal blood flow was closely associated with reduced central visual function. ${ }^{115}$ These findings reinforce the need for further evaluation of choroidal blood flow in patients with IRD. ${ }^{116}$

\section{Retinal oximetry}

Retinal oximetry devices have significantly advanced over the last decade, associated with improving reproducibility. ${ }^{117}$ Oxygen metabolism can be measured due to the different light absorption of oxy-haemoglobin and deoxy-haemoglobin. In IRD, outer retinal degeneration decreases the overall retinal oxygen requirement. Retinal loss also leads to increased diffusion of oxygen from the choroidal circulation into the inner layers, reducing the need for oxygen delivery from the retinal circulation, and ultimately causing increased venous saturation. ${ }^{118}$ Using retinal oximetry, oxygen saturation in retinal venules has been found to be significantly higher in patients with RCD than in controls, and the arteriovenous difference, lower. ${ }^{119} 120$ These findings were significantly associated with macular thickness and electrophysiology responses. ${ }^{121}$ Retinal oximetry's role in IRD requires further evaluation in large genotyped cohorts.

\section{Neuroimaging}

Magnetic resonance imaging (MRI) affords the ability to obtain high resolution structural images of the visual cortex and also sensitively record associated responses (functional MRI). ${ }^{122}$ Importantly, several MRI studies have described a degree of plasticity/remodelling following visual field defects in RCD or MD, and also with congenital visual impairment, including in ACHM. ${ }^{123-125}$ The remapping of the primary visual cortex (V1) consists of a shift of central retinal inputs to more peripheral locations in $\mathrm{V} 1$, and this phenomenon was found to be larger in patients with more constricted visual fields. ${ }^{125}$ However, it was noted that individuals with RCD did not have marked structural differences compared with controls (changes in white matter were mild); but in contrast, individuals with early-onset visual loss had thickened striate cortical and grey matter. ${ }^{125-128}$ It is of note that a child with ACHM who underwent gene therapy has been described who demonstrated cone-driven retinotopically organised signals in visual cortical areas, absent before the treatment. ${ }^{129}$ This raises the possibility of using MRI to measure ophthalmic gene therapy outcomes.

\section{Deep learning}

Imaging in medicine is now being aided by artificial intelligence-based algorithms that are intended to reduce errors and decrease analysing time. Deep learning consists of artificial neural networks that have self-learning algorithms based on large volumes of high-quality training data. ${ }^{130}$ While sufficiently large databases may be challenging to obtain for all IRD, this technology is already being applied to the IRD field.

Miere et al have used FAF images $(\mathrm{n}=389)$ to automatically classify IRD into the categories of Best disease, RCD, STGD, and controls, with an overall accuracy of $95 \% .{ }^{131}$ Arsalan $e t$ al chose instead CFP $(n=2160)$ to develop a network that segments the retina and detects pigment; with an accuracy of 99.5\%. ${ }^{132}$ Masumoto et al used 373 ultra-wide CFPs and FAF images to develop a platform that differentiates RCD from normal retinas, with a sensitivity and specificity of over $99 \% .{ }^{133}$ While, Fujinami-Yokokawa et al used OCT scans to create an approach that differentiates between retinal dystrophies secondary to pathogenic variants in ABCA4, RP1L1 and $E Y S$, with a mean accuracy of $90.9 \% .{ }^{134}$ Deep learning has also been used to binarize AF images from patients with RCD, and accurately identify and outline the hyperautofluorescent ring. This method showed statistically significant higher precision than subjective visual inspection. ${ }^{135}$ It is expected that deep learning algorithms will continue to improve and become integrated into high-definition technologies. This should help with more rapid accurate diagnosis and monitoring of disease, as well as facilitate trials, treatments and education.

\section{CONCLUSIONS}

Advancements in multimodal retinal imaging have transformed the practice of retinal genetics over the last 10 years, and no doubt will continue to evolve and expand over the next decade. These developments have shed light on disease mechanisms, allowed more timely diagnosis (helped to shorten the 'diagnostic odyssey') and earlier disease detection, prioritised genetic testing, facilitated more accurate advice on prognosis and more sensitive measurement of rate of change over time. They have also helped treatment development, cohort characterisation, trial design and outcome validation. Further improvements are anticipated, including with respect to ultrastructural imaging, metabolic imaging, improved structure-function overlays/correlation, and the establishment of artificial intelligence-mediated diagnostics to improve care and opportunities for patients with IRD.

Contributors All authors contributed to the design of this review article, literature review, manuscript preparation and review. The authors were responsible for all content and editorial decision.

Funding This work has been supported by grants from The Wellcome Trust (099173/Z/12/Z), the National Institute for Health Research Biomedical Research Centre at Moorfields Eye Hospital NHS Foundation Trust and UCL Institute of Ophthalmology, Moorfields Eye Charity, Retina UK and the Foundation Fighting Blindness (no specific grant/award number for the latter).

Disclaimer The views expressed are those of the authors and not necessarily those of the NHS, the NIHR or the Department of Health.

Competing interests The authors alone are responsible for the content and writing of this article. MM consults for MeiraGTX.

Patient consent for publication Not required.

Provenance and peer review Not commissioned; externally peer reviewed.

Open access This is an open access article distributed in accordance with the Creative Commons Attribution 4.0 Unported (CC BY 4.0) license, which permits others to copy, redistribute, remix, transform and build upon this work for any purpose, provided the original work is properly cited, a link to the licence is given, and indication of whether changes were made. See: https://creativecommons.org/ licenses/by/4.0/.

\section{ORCID iDs}

Malena Daich Varela http://orcid.org/0000-0003-4960-4510

Burak Esener http://orcid.org/0000-0002-9169-4989

Thales Antonio Cabral de Guimaraes http://orcid.org/0000-0002-7936-6851

Michalis Georgiou http://orcid.org/0000-0001-6397-8071

\section{REFERENCES}

1 Rahman N, Georgiou M, Khan KN, et al. Macular dystrophies: clinical and imaging features, molecular genetics and therapeutic options. Br J Ophthalmol 2020;104:451-60.

2 Gill JS, Georgiou M, Kalitzeos A, et al. Progressive cone and cone-rod dystrophies: clinical features, molecular genetics and prospects for therapy. Br J Ophthalmol 2019;103:711-20.

3 Kumaran N, Moore AT, Weleber RG, et al. Leber congenital amaurosis/early-onset severe retinal dystrophy: clinical features, molecular genetics and therapeutic interventions. Br J Ophthalmol 2017;101:1147-54.

4 Aboshiha J, Dubis AM, Carroll J, et al. The cone dysfunction syndromes. $\mathrm{Br}$ J Ophthalmol 2016;100:115-21. 
5 Hirji N, Aboshiha J, Georgiou M, et al. Achromatopsia: clinical features, molecular genetics, animal models and therapeutic options. Ophthalmic Genet 2018;39:149-57.

6 Hanany M, Rivolta C, Sharon D. Worldwide carrier frequency and genetic prevalence of autosomal recessive inherited retinal diseases. Proc Natl Acad Sci U SA 2020:117:2710-6.

7 Liew G, Michaelides M, Bunce C. A comparison of the causes of blindness certifications in England and Wales in working age adults (16-64 years), 1999-2000 with 2009-2010. BMJ Open 2014;4:e004015.

8 Galvin O, Chi G, Brady L, et al. The impact of inherited retinal diseases in the Republic of Ireland (Roi) and the United Kingdom (UK) from a cost-of-illness perspective. Clin Ophthalmol 2020;14:707-19.

9 Georgiou M, Fujinami K, Michaelides M. Retinal imaging in inherited retinal diseases. Ann Eye Sci 2020;5:25.

10 Daich Varela M, Cabral de Guimaraes TA, Georgiou M, et al. Leber congenital amaurosis/early-onset severe retinal dystrophy: current management and clinical trials. Br J Ophthalmol 2021. doi:10.1136/bjophthalmol-2020-318483. [Epub ahead of print: 12 Mar 2021].

11 Smith J, Ward D, Michaelides M, et al. New and emerging technologies for the treatment of inherited retinal diseases: a horizon scanning review. Eye 2015;29:1131-40.

12 Scholl HPN, Strauss RW, Singh MS, et al. Emerging therapies for inherited retinal degeneration. Sci Transl Med 2016;8:368rv6.

13 Bennett TJ, Barry CJ. Ophthalmic imaging today: an ophthalmic photographer's viewpoint - a review. Clin Exp Ophthalmol 2009;37:2-13.

14 Sarao V, Veritti D, Borrelli E, et al. A comparison between a white led confocal imaging system and a conventional flash fundus camera using chromaticity analysis. BMC Ophthalmol 2019;19:231

15 Bernardes R, Serranho P, Lobo C. Digital ocular fundus imaging: a review. Ophthalmologica 2011:226:161-81.

16 Salcedo-Villanueva G, Paciuc-Beja M, Villanueva-Mendoza C, et al. Progression of gyrate atrophy measured with ultra-wide-field imaging. Int Ophthalmol 2016:36:111-20

17 Kumar V, Samdani A, Chandra P, et al. Ultra-wide field imaging of retinal haemangioma in retinitis pigmentosa. Clin Exp Optom 2017;100:96-7.

18 Boulton M, Rózanowska M, Rózanowski B. Retinal photodamage. J Photochem Photobiol B 2001;64:144-61.

19 Delori FC, Dorey CK, Staurenghi G, et al. In vivo fluorescence of the ocular fundus exhibits retinal pigment epithelium lipofuscin characteristics. Invest Ophthalmol Vis Sci 1995;36:718-29.

20 Sparrow JR, Yoon KD, Wu Y, et al. Interpretations of fundus autofluorescence from studies of the bisretinoids of the retina. Invest Ophthalmo/ Vis Sci 2010;51:4351-7.

21 Keilhauer CN, Delori FC. Near-infrared autofluorescence imaging of the fundus: visualization of ocular melanin. Invest Ophthalmol Vis Sci 2006:47:3556-64.

22 Schmitz-Valckenberg S, Holz FG, Bird AC, et al. Fundus autofluorescence imaging: review and perspectives. Retina 2008:28:385-409.

23 Heiferman MJ, Fawzi AA. Discordance between blue-light autofluorescence and near-infrared autofluorescence in age-related macular degeneration. Retina 2016;36:\$137-46.

24 Schatz P, Preising M, Lorenz B, et al. Lack of autofluorescence in fundus albipunctatus associated with mutations in Rdh5. Retina 2010;30:1704-13.

25 Kumaran N, Georgiou M, Bainbridge JWB, et al. Retinal structure in RPE65Associated retinal dystrophy. Invest Ophthalmol Vis Sci 2020;61:47.

26 Paavo M, Carvalho JRL, Lee W, et al. Patterns and intensities of near-infrared and short-wavelength fundus autofluorescence in choroideremia probands and carriers. Invest Ophthalmol Vis Sci 2019;60:3752-61.

27 Saihan Z, Li Z, Rice J, et al. Clinical and biochemical effects of the E139K missense mutation in the TIMP3 gene, associated with Sorsby fundus dystrophy. Mo/ Vis 2009:15:1218-30.

28 Khabou H, Garita-Hernandez M, Chaffiol A, et al. Noninvasive gene delivery to foveal cones for vision restoration. JCI Insight 2018;3. doi:10.1172/jci.insight.96029. [Epub ahead of print: 25 Jan 2018].

29 Khan KN, Kasilian M, Mahroo OAR, et al. Early patterns of macular degeneration in ABCA4-Associated retinopathy. Ophthalmology 2018;125:735-46.

30 Liew G, Strong S, Bradley P, et al. Prevalence of cystoid macular oedema, epiretinal membrane and cataract in retinitis pigmentosa. Br J Ophthalmol 2019;103:1163-6.

31 Calvo-Maroto AM, Cerviño A. Spotlight on fundus autofluorescence. Clin Optom 2018:10:25-32.

32 von Rückmann A, Fitzke FW, Bird AC. Distribution of pigment epithelium autofluorescence in retinal disease state recorded in vivo and its change over time. Graefes Arch Clin Exp Ophthalmol 1999;237:1-9.

33 Lorenz B, Wabbels B, Wegscheider $\mathrm{E}$, et al. Lack of fundus autofluorescence to 488 nanometers from childhood on in patients with early-onset severe retinal dystrophy associated with mutations in RPE65. Ophthalmology 2004;111:1585-94.

34 Strauss RW, Ho A, Muñoz B, et al. The natural history of the progression of atrophy secondary to Stargardt disease (ProgStar) studies: design and baseline characteristics: ProgStar report No. 1. Ophthalmology 2016;123:817-28.
35 Kuehlewein L, Hariri AH, Ho A, et al. Comparison of manual and semiautomated fundus autofluorescence analysis of macular atrophy in Stargardt disease phenotype. Retina 2016;36:1216-21.

36 Strauss RW, Kong X, Ho A, et al. Progression of Stargardt disease as determined by fundus autofluorescence over a 12-month period. JAMA Ophthalmol 2019:137:1134-45.

37 Kong X, West SK, Strauss RW, et al. Progression of visual acuity and fundus autofluorescence in recent-onset stargardt disease: progStar study report \#4. Ophthalmol Retina 2017;1:514-23.

38 Strauss RW, Muñoz B, Ho A, et al. Progression of Stargardt disease as determined by fundus autofluorescence in the retrospective progression of Stargardt disease study (ProgStar report No. 9). JAMA Ophthalmol 2017;135:1232-41.

39 Kong X, Strauss RW, Cideciyan AV, et al. Visual acuity change over 12 months in the prospective progression of atrophy secondary to Stargardt disease (ProgStar) study: ProgStar report number 6. Ophthalmology 2017;124:1640-51.

40 Georgiou M, Robson AG, Singh N, et al. Deep phenotyping of PDE6C-Associated achromatopsia. Invest Ophthalmol Vis Sci 2019:60:5112-23.

41 Robson AG, Michaelides M, Saihan Z, et al. Functional characteristics of patients with retinal dystrophy that manifest abnormal parafoveal annuli of high density fundus autofluorescence; a review and update. Doc Ophthalmol 2008;116:79-89.

42 Lee TJ, Hwang JC, Chen RWS, et al. The role of fundus autofluorescence in late-onset retinitis pigmentosa (LORP) diagnosis. Ophthalmic Genet 2014;35:170-9

43 Robson AG, Michaelides M, Luong VA, et al. Functional correlates of fundus autofluorescence abnormalities in patients with RPGR or RIMS1 mutations causing cone or cone rod dystrophy. Br J Ophthalmol 2008:92:95-102.

44 Tee JJL, Kalitzeos A, Webster AR, et al. Quantitative analysis of hyperautofluorescent rings to characterize the natural history and progression in RPGR-associated retinopathy. Retina 2018:38:2401-14.

45 Duncker T, Tabacaru MR, Lee W, et al. Comparison of near-infrared and shortwavelength autofluorescence in retinitis pigmentosa. Invest Ophthalmol Vis Sci 2013:54:585-91.

46 Cicinelli MV, Marchese A, Bordato A, et al. Reviewing the role of ultra-widefield imaging in inherited retinal dystrophies. Ophthalmol Ther 2020;9:249-63.

47 Georgiou M, Kane T, Tanna P, et al. Prospective cohort study of childhoodonset Stargardt disease: fundus autofluorescence imaging, progression, comparison with adult-onset disease, and disease symmetry. Am J Ophthalmol 2020;211:159-75.

48 Hariri AH, Velaga SB, Girach A, et al. Measurement and reproducibility of preserved ellipsoid zone area and preserved retinal pigment epithelium area in eyes with choroideremia. Am J Ophthalmol 2017:179:110-7.

49 Vujosevic S, Toma C, Nucci P, et al. Quantitative color fundus autofluorescence in patients with diabetes mellitus. J Clin Med 2020;10. doi:10.3390/jcm10010048. [Epub ahead of print: 25 Dec 2020].

50 Borrelli E, Lei J, Balasubramanian S, et al. Green emission fluorophores in eyes with atrophic age-related macular degeneration: a colour fundus autofluorescence pilot study. Br J Ophthalmol 2018;102:827-32.

51 Wolf-Schnurrbusch UEK, Wittwer VV, Ghanem R, et al. Blue-light versus green-light autofluorescence: lesion size of areas of geographic atrophy. Invest Ophthalmo/ Vis Sci 2011;52:9497-502.

52 Cideciyan AV, Swider M, Aleman TS, et al. Reduced-illuminance autofluorescence imaging in ABCA4-associated retinal degenerations. J Opt Soc Am A Opt Image Sci Vis 2007:24:1457-67.

53 Strauss RW, Muñoz B, Jha A, et al. Comparison of short-wavelength ReducedIlluminance and conventional autofluorescence imaging in Stargardt macular dystrophy. Am J Ophthalmol 2016;168:269-78.

54 Bernstein P, Dysli C, Fischer J. Fluorescence lifetime imaging ophthalmoscopy (FLIO), 2019: 213-35.

55 Andersen KM, Sauer L, Gensure RH, et al. Characterization of retinitis pigmentosa using fluorescence lifetime imaging ophthalmoscopy (FLIO). Trans/ Vis Sci Technol 2018;7:20.

56 Huang D, Swanson EA, Lin CP, et al. Optical coherence tomography. Science 1991:254:1178-81.

57 Gabriele ML, Wollstein G, Ishikawa H, et al. Optical coherence tomography: history, current status, and laboratory work. Invest Ophthalmol Vis Sci 2011;52:2425-36.

58 de Boer JF, Cense B, Park BH, et al. Improved signal-to-noise ratio in spectraldomain compared with time-domain optical coherence tomography. Opt Lett 2003:28:2067-9.

59 Lim LS, Cheung G, Lee SY. Comparison of spectral domain and swept-source optical coherence tomography in pathological myopia. Eye 2014:28:488-91.

60 Vira J, Marchese A, Singh RB, et al. Swept-source optical coherence tomography imaging of the retinochoroid and beyond. Expert Rev Med Devices 2020;17:413-26.

61 Syed R, Sundquist SM, Ratnam K, et al. High-resolution images of retinal structure in patients with choroideremia. Invest Ophthalmol Vis Sci 2013;54:950-61.

62 Gerth-Kahlert C, Tiwari A, Hanson JVM, et al. C2Orf71 mutations as a frequent cause of autosomal-recessive retinitis pigmentosa: clinical analysis and presentation of 8 novel mutations. Invest Ophthalmol Vis Sci 2017:58:3840-50.

63 Sieving PA, MacDonald IM. X-Linked congenital retinoschisis, 1993. 
64 Jacobson SG, Cideciyan AV, Aleman TS, et al. Crumbs homolog 1 (CRB1) mutations result in a thick human retina with abnormal lamination. Hum Mol Genet 2003;12:1073-8.

65 Milam AH, Li ZY, Fariss RN. Histopathology of the human retina in retinitis pigmentosa. Prog Retin Eye Res 1998;17:175-205.

66 Ko TH, Fujimoto JG, Duker JS, et al. Comparison of ultrahigh- and standardresolution optical coherence tomography for imaging macular hole pathology and repair. Ophthalmology 2004;111:2033-43.

67 Fischer MD, Fleischhauer JC, Gillies MC, et al. A new method to monitor visual field defects caused by photoreceptor degeneration by quantitative optical coherence tomography. Invest Ophthalmo/ Vis Sci 2008;49:3617-21.

68 Aizawa S, Mitamura Y, Hagiwara A, et al. Changes of fundus autofluorescence, photoreceptor inner and outer segment junction line, and visual function in patients with retinitis pigmentosa. Clin Exp Ophthalmol 2010;38:597-604.

69 Hara A, Nakazawa M, Saito M, et al. The qualitative assessment of optical coherence tomography and the central retinal sensitivity in patients with retinitis pigmentosa. PLoS One 2020;15:e232700.

70 Hariri AH, Zhang HY, Ho A, et al. Quantification of ellipsoid zone changes in retinitis pigmentosa using en face spectral Domain-Optical coherence tomography. JAMA Ophthalmol 2016;134:628-35.

71 Tee JJL, Yang Y, Kalitzeos A, et al. Natural history study of retinal structure, progression, and symmetry using Ellipzoid zone metrics in RPGR-associated retinopathy. Am J Ophthalmol 2019;198:111-23.

72 Tanna P, Georgiou M, Strauss RW, et al. Cross-sectional and longitudinal assessment of the ellipsoid zone in childhood-onset Stargardt disease. Trans/ Vis Sci Technol 2019;8:1.

73 Alabduljalil T, Patel RC, Alqahtani AA, et al. Correlation of outer retinal degeneration and Choriocapillaris loss in Stargardt disease using en face optical coherence tomography and optical coherence tomography angiography. Am J Ophthalmol 2019:202:79-90.

74 Weng J, Mata NL, Azarian SM, et al. Insights into the function of rim protein in photoreceptors and etiology of Stargardt's disease from the phenotype in $A B C R$ knockout mice. Cell 1999:98:13-23.

75 Son G, Lee S, Kim YJ, et al. Correlation between visual function and structural characteristics of the macula in advanced retinitis pigmentosa. Ophthalmologica 2019;242:22-30

76 Ramachandran R, Zhou L, Locke KG, et al. A comparison of methods for tracking progression in X-linked retinitis pigmentosa using frequency domain OCT. Trans/Vis Sci Technol 2013:2:5.

77 Langlo CS, Patterson EJ, Higgins BP, et al. Residual foveal cone structure in CNGB3associated achromatopsia. Invest Ophthalmol Vis Sci 2016:57:3984-95.

78 Georgiou M, Litts KM, Kalitzeos A, et al. Adaptive optics retinal imaging in CNGA3Associated achromatopsia: retinal characterization, Interocular symmetry, and intrafamilial variability. Invest Ophthalmol Vis Sci 2019;60:383-96.

79 Mastey RR, Georgiou M, Langlo CS, et al. Characterization of retinal structure in ATF6-Associated achromatopsia. Invest Ophthalmol Vis Sci 2019;60:2631-40.

80 Velaga SB, Nittala MG, Jenkins D, et al. Impact of segmentation density on spectral domain optical coherence tomography assessment in Stargardt disease. Graefes Arch Clin Exp Ophthalmol 2019;257:549-56.

81 Ervin A-M, Strauss RW, Ahmed Ml, et al. A workshop on measuring the progression of atrophy secondary to Stargardt disease in the ProgStar studies: findings and lessons learned. Trans/ Vis Sci Technol 2019:8:16.

82 Kong X, Ho A, Munoz B, et al. Reproducibility of measurements of retinal structural parameters using optical coherence tomography in Stargardt disease. Trans/ Vis Sci Technol 2019:8:46.

83 Strauss RW, Muñoz B, Wolfson Y, et al. Assessment of estimated retinal atrophy progression in Stargardt macular dystrophy using spectral-domain optical coherence tomography. Br J Ophthalmol 2016;100:956-62.

84 Lam BL, Davis JL, Gregori NZ, et al. Choroideremia gene therapy phase 2 clinical trial: 24-month results. Am J Ophthalmol 2019;197:65-73.

85 Spaide RF, Fujimoto JG, Waheed NK, et al. Optical coherence tomography angiography. Prog Retin Eye Res 2018;64:1-55.

86 Inooka D, Ueno S, Kominami T, et al. Quantification of macular microvascular changes in patients with retinitis pigmentosa using optical coherence tomography angiography. Invest Ophthalmol Vis Sci 2018;59:433-8.

87 Sugahara M, Miyata M, Ishihara K, et al. Optical coherence tomography angiography to estimate retinal blood flow in eyes with retinitis pigmentosa. Sci Rep 2017;7:46396.

88 Miyata M, Oishi A, Hasegawa T, et al. Concentric Choriocapillaris flow deficits in retinitis pigmentosa detected using wide-angle Swept-Source optical coherence tomography angiography. Invest Ophthalmo/ Vis Sci 2019;60:1044-9.

89 Léveillard T, Sahel J-A. Metabolic and redox signaling in the retina. Cell Mol Life Sci 2017;74:3649-65

90 Cetin EN, Parca O, Akkaya HS, et al. Association of retinal biomarkers and choroidal vascularity index on optical coherence tomography using binarization method in retinitis pigmentosa. Graefes Arch Clin Exp Ophthalmol 2020;258:23-30.

91 Lin R, Shen M, Pan D, et al. Relationship between cone loss and microvasculature change in retinitis pigmentosa. Invest Ophthalmol Vis Sci 2019;60:4520-31.
92 Liu R, Lu J, Liu Q, et al. Effect of choroidal vessel density on the ellipsoid zone and visual function in retinitis pigmentosa using optical coherence tomography angiography. Invest Ophthalmol Vis Sci 2019;60:4328-35.

93 Georgiou M, Kalitzeos A, Patterson EJ, et al. Adaptive optics imaging of inherited retinal diseases. Br J Ophthalmol 2018;102:1028-35.

94 Genead MA, Fishman GA, Rha J, et al. Photoreceptor structure and function in patients with congenital achromatopsia. Invest Ophthalmol Vis Sci 2011:52:7298-308

95 Sundaram V, Wilde C, Aboshiha J, et al. Retinal structure and function in achromatopsia: implications for gene therapy. Ophthalmology 2014;121:234-45.

96 Foote KG, Wong JJ, Boehm AE, et al. Comparing cone structure and function in rho- and RPGR-associated retinitis pigmentosa. Invest Ophthalmol Vis SC 2020;61:42.

97 Litts KM, Georgiou M, Langlo CS, et al. Interocular symmetry of foveal cone topography in congenital achromatopsia. Curr Eye Res 2020:45:1257-64.

98 Litts KM, Cooper RF, Duncan JL, et al. Photoreceptor-based biomarkers in AOSLO retinal imaging. Invest Ophthalmol Vis Sci 2017:58:BI0255.

99 Duncan JL, Zhang Y, Gandhi J, et al. High-resolution imaging with adaptive optics in patients with inherited retinal degeneration. Invest Ophthalmol Vis Sci 2007:48:3283-91.

100 Nakatake S, Murakami Y, Funatsu J, et al. Early detection of cone photoreceptor cell loss in retinitis pigmentosa using adaptive optics scanning laser ophthalmoscopy. Graefes Arch Clin Exp Ophthalmol 2019;257:1169-81.

101 Ueda-Consolvo T, Ozaki H, Nakamura T, et al. The association between cone density and visual function in the macula of patients with retinitis pigmentosa. Graefes Arch Clin Exp Ophthalmol 2019:257:1841-6.

102 Georgiou M, Singh N, Kane T, et al. Photoreceptor structure in GNAT2-Associated achromatopsia. Invest Ophthalmol Vis Sci 2020;61:40.

103 Tanna P, Kasilian M, Strauss R, et al. Reliability and repeatability of cone density measurements in patients with Stargardt disease and RPGR-associated retinopathy. Invest Ophthalmol Vis Sci 2017;58:3608-15.

104 Georgiou M, Litts KM, Singh N, et al. Intraobserver Repeatability and Interobserver Reproducibility of Foveal Cone Density Measurements in CNGA3- and CNGB3Associated Achromatopsia. Trans/ Vis Sci Technol 2020;9:37

105 Strauss RW, Dubis AM, Cooper RF, et al. Retinal architecture in RGS9- and R9APAssociated retinal dysfunction (Bradyopsia). Am J Ophthalmol 2015;160:1269-75.

106 Ratnam K, Carroll J, Porco TC, et al. Relationship between foveal cone structure and clinical measures of visual function in patients with inherited retinal degenerations. Invest Ophthalmol Vis Sci 2013:54:5836-47.

107 Hillmann D, Spahr H, Pfäffle C, et al. In vivo optical imaging of physiological responses to photostimulation in human photoreceptors. Proc Natl Acad Sci U S A 2016:113:13138-43.

108 Bennett N. Light-Induced interactions between rhodopsin and the GTP-binding protein. relation with phosphodiesterase activation. Eur J Biochem 1982;123:133-9.

109 Srinivasan VJ, Dubra A. Noninvasive imaging of the photoreceptor mosaic response to light stimulation. Proc Natl Acad Sci U S A 2016;113:12902-3.

110 Srinivasan VJ, Chen Y, Duker JS, et al. In vivo functional imaging of intrinsic scattering changes in the human retina with high-speed ultrahigh resolution OCT. Opt Express 2009;17:3861-77

111 Zhang F, Kurokawa K, Lassoued A, et al. Cone photoreceptor classification in the living human eye from photostimulation-induced phase dynamics. Proc Natl Acad Sci USA 2019;116:7951-6.

112 Bedggood P, Metha A. Optical imaging of human cone photoreceptors directly following the capture of light. PLoS One 2013:8:e79251

113 Kim T-H, Wang B, Lu Y, et al. Functional optical coherence tomography enables in vivo optoretinography of photoreceptor dysfunction due to retinal degeneration. Biomed Opt Express 2020;11:5306-20.

114 Sugiyama T, Araie M, Riva CE, et al. Use of laser speckle flowgraphy in ocular blood flow research. Acta Ophthalmol 2010:88:723-9.

115 Murakami Y, Ikeda Y, Akiyama M, et al. Correlation between macular blood flow and central visual sensitivity in retinitis pigmentosa. Acta Ophthalmol 2015;93:e644-8.

116 Murakami Y, Funatsu J, Nakatake S, et al. Relations among foveal blood flow, Retinal-Choroidal structure, and visual function in retinitis pigmentosa. Invest Ophthalmol Vis Sci 2018:59:1134-43.

117 Stefánsson E, Olafsdottir OB, Eliasdottir TS, et al. Retinal oximetry: metabolic imaging for diseases of the retina and brain. Prog Retin Eye Res 2019:70:1-22.

118 Eysteinsson T, Hardarson SH, Bragason D, et al. Retinal vessel oxygen saturation and vessel diameter in retinitis pigmentosa. Acta Ophthalmol 2014;92:449-53.

119 Battu R, Mohan A, Khanna A, et al. Retinal oxygen saturation in retinitis pigmentosa and macular dystrophies in asian-Indian eyes. Invest Ophthalmol Vis Sci 2015; 56:2798-802.

120 Türksever C, Valmaggia C, Orgül S, et al. Retinal vessel oxygen saturation and its correlation with structural changes in retinitis pigmentosa. Acta Ophthalmol 2014:92:454-60.

121 Todorova MG, Türksever C, Schötzau A, et al. Metabolic and functional changes in retinitis pigmentosa: comparing retinal vessel oximetry to full-field electroretinography, electrooculogram and multifocal electroretinography. Acta Ophthalmol 2016;94:e231-41. 
122 Castaldi E, Cicchini GM, Falsini B, et al. Residual visual responses in patients with retinitis pigmentosa revealed by functional magnetic resonance imaging. Trans/ Vis Sci Technol 2019;8:44.

123 Castaldi E, Lunghi C, Morrone MC. Neuroplasticity in adult human visual cortex. Neurosci Biobehav Rev 2020;112:542-52.

124 Sanda N, Cerliani L, Authié CN, et al. Visual brain plasticity induced by central and peripheral visual field loss. Brain Struct Funct 2018;223:3473-85.

125 Ferreira S, Pereira AC, Quendera B, et al. Primary visual cortical remapping in patients with inherited peripheral retinal degeneration. Neuroimage Clin 2017;13:428-38.

126 Cunningham SI, Shi Y, Weiland JD, et al. Feasibility of structural and functional MRI acquisition with Unpowered implants in argus II retinal prosthesis patients: a case study. Trans/ Vis Sci Technol 2015;4:6.

127 Aguirre GK, Butt $\mathrm{OH}$, Datta R, et al. Postretinal structure and function in severe congenital photoreceptor blindness caused by mutations in the GUCY2D gene. Invest Ophthalmol Vis Sci 2017;58:959-73.

128 Hofstetter S, Sabbah N, Mohand-Said S, et al. The development of white matter structural changes during the process of deterioration of the visual field. Sci Rep 2019;9:2085.
129 Farahbakhsh M, Anderson EJ, Rider A. A demonstration of cone function plasticity after gene therapy in achromatopsia. medRxiv 2020. doi:10.1101/2020.12.16.20246710

130 Schmidt-Erfurth U, Sadeghipour A, Gerendas BS, et al. Artificial intelligence in retina. Prog Retin Eye Res 2018;67:1-29.

131 Miere A, Le Meur T, Bitton K, et al. Deep learning-based classification of inherited retinal diseases using fundus autofluorescence. J Clin Med 2020;9. doi:10.3390/ jcm9103303. [Epub ahead of print: 14 Oct 2020].

132 Arsalan M, Baek NR, Owais M, et al. Deep Learning-Based detection of pigment signs for analysis and diagnosis of retinitis pigmentosa. Sensors 2020;20. doi:10.3390/s20123454. [Epub ahead of print: 18 Jun 2020].

133 Masumoto H, Tabuchi H, Nakakura S, et al. Accuracy of a deep convolutional neural network in detection of retinitis pigmentosa on ultrawide-field images. PeerJ 2019;7:e6900.

134 Fujinami-Yokokawa Y, Pontikos N, Yang L, et al. Prediction of causative genes in inherited retinal disorders from spectral-domain optical coherence tomography utilizing deep learning techniques. J Ophthalmol 2019;2019:1-7.

135 Hashimoto $Y$, Inoue T, Ono T, et al. A novel method for the objective identification of Hyperautofluorescent ring in retinitis pigmentosa using Binarization processing. Trans/ Vis Sci Technol 2019;8:20. 